\title{
Noise-reducing designs of machines and structures
}

\author{
N TANDON \\ ITMMEC, Indian Institute of Technology, New Delhi 110016, India \\ e-mail: ntandon@itmmec.iitd.ernet.in
}

\begin{abstract}
It is advisable and better to consider noise control measures at the design stage itself rather than after the product is manufactured and installed. The present paper describes some of the control measures that can be incorporated during the design of machines and structures. Vibration control, barriers and enclosures can be used for noise control and should be considered at the design stage. Acoustical materials which can be used for noise control purpose have been described. Some examples of noise-reducing designs are also given.
\end{abstract}

Keywords. Noise control; vibration control; barriers to noise; noise-free enclosures; acoustical materials; noise-reducing designs.

\section{Introduction}

Industrialisation, together with the needs of our modern society for various machines for human comfort, fast travel and appliances for routine jobs in homes and offices, has led to an increase in the levels of noise pollution almost everywhere. The harmful effects of noise are well-known. Exposure to high noise levels can cause hearing loss. Noise can also result in other ill effects such as general annoyance, loss of sleep, headache, stress, constriction of blood vessels and deterioration in work performance. Thus it is important to reduce noise levels as much as possible. Various techniques for noise control are available, and it is advisable to consider noise control methods at the design stage itself. Some noise control measures that can be adopted by the designer at the design stage are described in this article. In many situations the methods described can also be applied at the development stage or even later to control noise levels.

\section{Vibration control}

Most noise sources (except for aerodynamic noise) are associated with vibrating surfaces. Hence the control of vibration is an important part of any noise control programme. Vibration control can be achieved by isolation, damping and by avoiding resonance in structures and machine parts. 


\subsection{Vibration isolation}

High noise can result because of vibration transmission from a source to some structure which is a better noise radiator than the source itself. Hence vibration isolation is an important consideration while designing machines and their mountings. Transmission of vibrations from one structure to another can be reduced by the use of resilient elements between them. Resilient elements which may be visualized as springs, are called vibration isolators. Vibration isolation can be understood from the analysis of an ideal, linear, singledegree of freedom system in which the isolator is represented by the parallel combination of massless spring and damper. The transmissibility, $T$, of this system is defined as the ratio of the amplitudes of the mass displacement to that of the disturbing motion of the support and is given as

$$
T=\left\{\left[1+(2 \zeta r)^{2}\right] /\left[\left(1-r^{2}\right)^{2}+(2 \zeta r)^{2}\right]\right\}^{1 / 2},
$$

where $\zeta$ is the damping ratio and $r$ is the ratio of the excitation frequency to the natural frequency of the spring mass system. The isolation range of this system is where $r>\sqrt{2}$ i.e. where $T$ is less than unity. In other words, for an isolator to be successful, its stiffness must be such that the mounted resonance frequency is less than 0.7 times the minimum forcing frequency. The isolation system should be carefully designed because if the value of $r$ is near 1.0, then instead of attenuation, the vibrations will be amplified.

Such a single-degree of freedom model provides some insight into the behaviour of an isolation system, but does not account for many aspects of realistic installations. Real springs are not massless and the supported masses generally move not only vertically but also horizontally and also tend to rock. Different types of practical isolators are available commercially. Most commercial isolators incorporate metallic or elastomeric resilient elements. Metallic elements are usually in the form of coil springs or flexural configurations such as leaf springs. Isolators with elastomeric elements normally have these elements bonded or otherwise attached to support plates to provide convenient means of fastening to other components. An elastomeric element may be used in shear, torsion, compression modes or a combination of these. The pneumatic or air spring isolators use the principle of compressibility of confined volumes of air. In active isolation systems, the vibration of the structure to be protected is sensed by a transducer whose output is used to drive an actuator that acts on the structure so as to reduce its vibration.

\subsection{Vibration damping}

Noise characteristics of structures are determined by mass, stiffness and damping. Whereas mass and stiffness are associated with the storage of kinetic and strain energy respectively, damping relates to the dissipation of energy. The increased damping results in faster decay of unforced vibrations and reduced amplitude at resonance of structures subject to steady excitations. The ratio of the energy dissipated per cycle to the energy present in the system is called the specific damping capacity, $\psi$. The loss factor, $\eta$, is often used to specify material damping and the relationship between these quantities is:

$$
\eta=(\psi / 2 \pi)=2 \zeta
$$

The specific damping capacities of mild steel, austenitic flake graphite cast iron and nylon 12 are $1.5,7$ and 31 respectively. 
It is possible to introduce additional damping to a structure by means of damping layers of viscoelastic material. The damping layer may be either unconstrained or constrained. In the case of unconstrained layers, the damping material which is attached to the metal or alloy base, is subjected to longitudinal extensions and contractions. In most cases, optimum damping is achieved when the thickness of the damping material is several times that of the base thickness. Constrained damping layers are more effective. In this case the damping material is coated with a thin sheet of metal. The damping layer is subjected to shear deformation, as it is sandwiched between two sheets. Sandwich sheets with the damping material in between two steel sheets are commercially available.

\subsection{Avoiding resonances}

Natural frequencies of the structures should be estimated at the design stage and, if required, the structure should be stiffened so that the natural frequencies of the structure are usually increased above the frequency of the excitation forces. The natural frequencies can be estimated analytically or by finite element analysis. In some cases, it may not be possible to avoid resonances, especially where impact forces are encountered such as in IC engine combustion process. In such cases, the application of damping treatment is helpful in reducing noise generation from structures.

The importance of resonance consideration at the design stage is illustrated in the following example where its non-consideration resulted in high noise generation from a table fan (Tandon \& Nakra 1994). The major noise source in the table fan (without blades) was located by measuring sound intensity over a grid layer on the front side of the fan as shown in figure 1. The 3-dimensional intensity map obtained from these measurements (figure 2) indicated that in addition to the high intensity levels obtained in the region of the fan motor, unexpected and higher intensity levels were obtained in the region

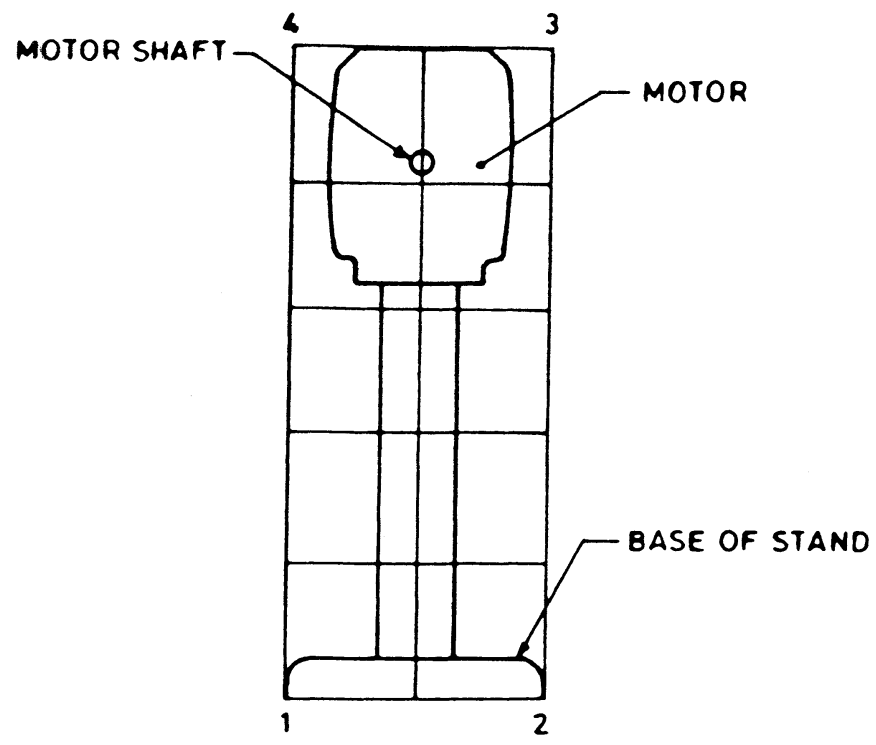

Figure 1. Sound intensity measurement grid on the front side of the table fan. 


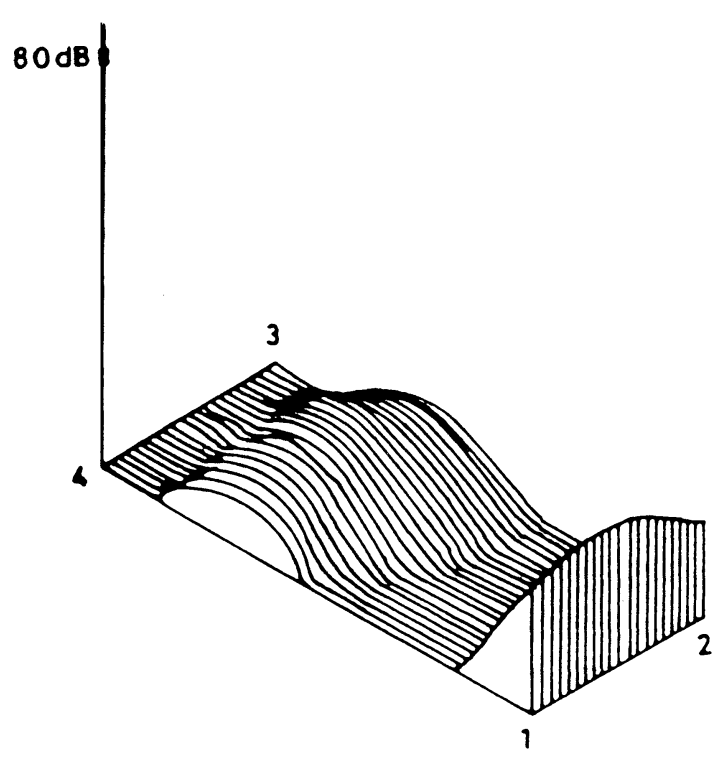

Figure 2. 3-dimensional sound intensity map of the front side of the table fan.

corresponding to the base of the fan stand indicating that the base was radiating high noise levels. The intensity spectrum showed main peaks at 524, 620, 720 and $816 \mathrm{~Hz}$. Natural frequencies of the fan stand were measured by the hammer test and some of the peaks were very close to these frequencies indicating that the cause of high noise was the excitation of the natural frequencies of the stand. The excitation forcing frequencies were traced to the magnetic noise of the fan motor. Magnetic noise frequencies depend upon the number of rotor slots, rotor speed and the line frequency because of slot harmonic force field. These frequencies, calculated at the measured rotor speed of $1430 \mathrm{rpm}$, are given in table 1 , where $k$ is no. of harmonics $(1,2, \ldots), R$ is no. of rotor slots, $n$ is rotor speed (rps), $b$ is the positive integer and $f$ is line frequency $(50 \mathrm{~Hz})$.

When the natural frequency of the fan stand was changed by increasing the mass of the base, the noise reduced by about $10 \mathrm{~dB}(\mathrm{~A})$.

\section{Barriers}

Barriers can be designed between noise sources and receivers. They block the line of sight between source and receiver as shown in figure 3. In the case of long barriers, the noise from the source is diffracted (bent) downwards after reaching the top of the barrier. The

Table 1. Magnetic noise excitation frequencies of the table fan motor at $1430 \mathrm{rpm}$.

\begin{tabular}{ll}
\hline Magnetic noise & \multicolumn{1}{c}{ Frequency $(\mathrm{Hz})$} \\
\hline$k R n$ & $428.4,856.8, \ldots \ldots$ \\
$k R n+2 b f$ & $528.4,628.4,728.4,828.4, \ldots$ \\
$k R n-2 b f$ & $328.4,228.4,128.4, \ldots \ldots$ \\
& $756.8,656.8,556.8, \ldots \ldots$ \\
\hline
\end{tabular}




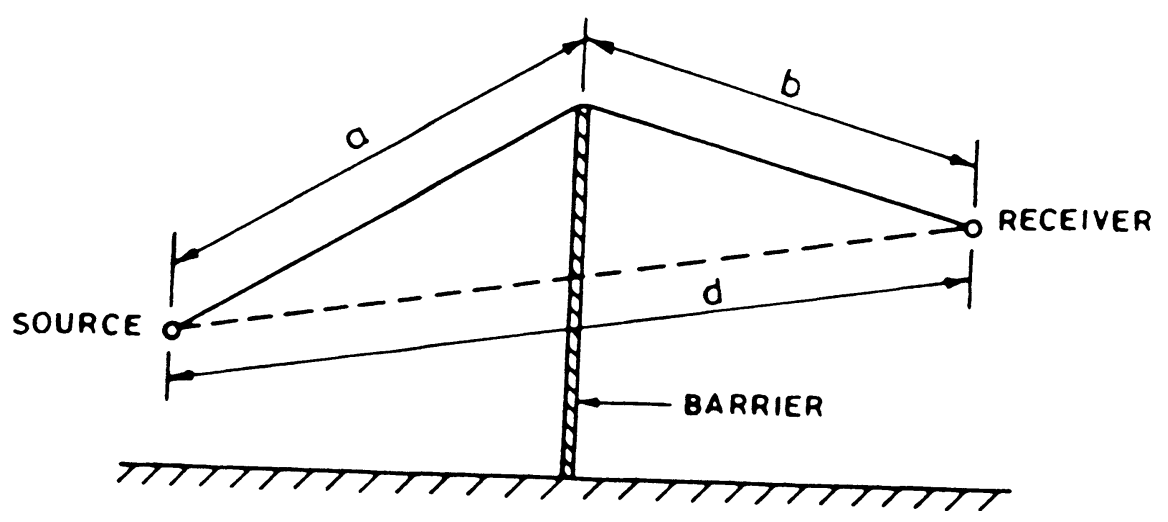

Figure 3. A barrier.

Fresnel number, $N$ of the barrier is defined as,

$$
\begin{aligned}
N= & \pm(2 / \lambda)(a+b-d) \\
& + \text { when receiver is in shadow zone, } \\
& - \text { when receiver is in bright zone. }
\end{aligned}
$$

The barrier attenuation for a point source can be calculated as:

$$
\begin{aligned}
\text { attenuation } & =20 \log \frac{(2 \pi N)^{1 / 2}}{\tanh (2 \pi N)^{1 / 2}}+5, \quad \text { for } N \geq-0.2 \\
& =0, \quad \text { otherwise. }
\end{aligned}
$$

The barrier attenuation equation assumes that diffracted sound dominates at the receiver and the sound passing through the barrier must be negligible compared to diffracted sound. This requires that the barrier be free of holes and any gap between it and the ground.

It is very common to use traffic noise barriers to protect people living near busy roads. In recent years, such barriers of different shapes have been studied by Watt et al (1994). T- and Y-shape barriers have been used with and without absorbent material lining. The acoustic performance of such barriers has been reported to be better by about 1.5 to $3 \mathrm{~dB}$ than that of the straight wall construction. Double barriers have also been used.

\section{Enclosures}

Enclosure is a commonly used form of noise control at the workplace. It is also possible to incorporate enclosures into the design for machines or their parts. In an enclosure, any pipes from the machine should not be rigidly attached to the enclosure, but should be supported by vibration isolating hangers. Air gaps need to be minimized. Ducts for fresh air and exhaust gases should be adequately silenced. The insides of the panels of enclosures are lined with absorbent materials to avoid build-up of noise due to reflections.

While designing the enclosure, two types of enclosure resonances are taken into account. The first is the mechanical resonance of the enclosure panels and the other is the acoustic resonance of the air-space between the enclosed machine and the enclosure walls. The first few panel resonance frequencies of the enclosure should not be in the frequency range in 
Table 2. Sound pressure levels of generator.

\begin{tabular}{|c|c|c|c|c|c|c|}
\hline & \multicolumn{6}{|c|}{ Sound pressure level $(\mathrm{dB}(\mathrm{A}))$} \\
\hline & \multicolumn{2}{|c|}{ Front } & \multicolumn{2}{|c|}{ Back } & \multicolumn{2}{|c|}{ Top } \\
\hline & No load & $800 \mathrm{~W}$ load & No load & $800 \mathrm{~W}$ load & No load & $800 \mathrm{~W}$ load \\
\hline Without enclosure & 85.5 & 87.0 & 82.0 & 82.5 & 82.0 & 82.5 \\
\hline With partial enclosure & 82.0 & 84.0 & 80.0 & 81.0 & 77.0 & 78.0 \\
\hline
\end{tabular}

which sound attenuation is desired. If the sound source radiates predominantly high frequency noise, then an enclosure with low frequency panels is recommended, implying a massive enclosure. On the other hand, if the sound radiation is predominantly low frequency in nature, then an enclosure with a high resonance frequency is desirable, implying a stiff but not massive enclosure. Inside the enclosure a reverberant sound field is produced in addition to the noise from the source. Also, acoustic resonances occur at the standing wave frequency given by (Ubhe 1996)

$$
f=c / 2 d,
$$

where $c$ is the speed of sound and $d$ is the distance between source and panel. To suppress acoustic resonances, absorbent material can be used on the inside of the enclosure. Absorbent material reduces the amplitude of the standing waves, and the layer of such material should be about half the thickness of the air space to damp out the resonance considerably.

An example of a partial enclosure designed for a portable generator set as studied by Ubhe (1996) is as below. The noise spectra of the generator indicated that low frequency noise was predominant, so an enclosure with stiff panels was designed. Sandwich-type walls were used to get high stiffness. A uniform layer of foam of $25 \mathrm{~mm}$ thickness was applied on the inside of the enclosure panels. The enclosure covered about two-thirds of the generator set. The sound pressure levels measured at $1 \mathrm{~m}$ distance from some of the sides of the generator with and without enclosures are given in table 2 .

A reduction of 3 to $5 \mathrm{~dB}(\mathrm{~A})$ was obtained. In full enclosures with minimum openings, insertion losses of more than $20 \mathrm{~dB}(\mathrm{~A})$ can easily be obtained.

\section{Acoustical materials}

Acoustical materials for the purpose of noise reduction are materials that absorb sound, reflect sound, or dampen vibrations. Acoustical properties of the materials used in different situations should be taken into account during the design stage. Wherever possible, quieter plastics should be used in place of metal components. Cast iron has better material damping than steel. Acoustical materials usually convert some of the sound energy which strikes them into thermal energy.

Materials which reflect or insulate noise are used as barriers. While any material will reflect some of the noise reaching it, only dense and airtight materials are really effective in blocking noise. Some good insulating materials are concrete, thick steel sheets, glass, lead and thick PVC sheets. Sound-absorbing materials are used to reduce noise levels within spaces, to prevent reflections from surfaces and to control reverberation within spaces. Unfortunately, most good noise absorbents are poor noise barriers. They are usually porous and lightweight. Foam, fibreglass, cork and mineral wool are common sound-absorbing 
materials. Metal wools can be used in situations where high temperatures are encountered. Materials used to damp vibrations are usually viscoelastic materials such as filled bitumens, specially formulated elastomers and some types of polymer plastics.

\section{Noise reduction based upon the knowledge of behaviour of sound}

Simple changes in production processes or factory layout, keeping in view the behaviour of noise, can result in noise reduction. Sudden and large changes in force, pressure or speed lead to high noise. Thus, if a metal strip fixed in a vice is bent with pliers instead of by hammering, much less noise is produced. Similarly, using nails driven by hammering, instead of wood screws or nut bolts, is a more noisy process. High frequency sound is easy to reflect. Therefore screens to reflect such noise away from the operator can be used between machines generating high frequency noises and their operators. Low height of drop gives less noise. Thus the height of drop of scrap metal pieces in a bin should be reduced (by raising the bin position). Indoor screen barriers should be combined with absorbent ceilings. Air flows over cavities should be avoided in the air stream to reduce turbulence noise and pipe bends should be smooth without any sharp corners.

Factory layouts should be planned in such a way that noisy machines are kept away from reflecting walls as much as possible. The worst position is near a corner, i.e. near three reflecting surfaces. The noisiest machines can be placed together and the less noisy ones in a different area. This way one noisy and one quieter area will be obtained. The noisy area will probably not become as noisy as one may imagine because of the fact that if two sources are generating say $100 \mathrm{~dB}$ noise, then their combined level will be only $103 \mathrm{~dB}$. The combined level of 10 such sources will be $110 \mathrm{~dB}$ only. Subjectively, an increase of 3 $\mathrm{dB}$ is just noticeable, $5 \mathrm{~dB}$ is clearly noticeable and $10 \mathrm{~dB}$ sounds twice as loud.

\section{Some typical noise-reducing designs}

\subsection{Piston slap noise in IC engines}

An important mechanical noise source in reciprocating internal combustion engines is usually piston slap. This noise can be reduced by modifying the kinematics of the piston by offsetting the piston pin. A small (about $0.5-2 \mathrm{~mm}$ ) lateral displacement of the piston pin away from the piston axis is provided without altering its location in the vertical direction. With this, advanced timing of the piston slap is achieved. Piston slap no longer takes place shortly after top dead centre (TDC) under the sudden burst of combustion, but rather just before TDC under the compression pressure which builds up relatively slowly (Rohrle 1975). Another way of reducing piston slap noise is by reducing the clearance between piston and cylinder. The noise reduction achieved by reducing this clearance for an engine of a two-wheeler scooter is given in table 3 (Tandon et al 1997).

In general, the danger of scuffing/seizure does not permit reduction in piston clearances without using light alloy cylinders or positive oil-cooling of the piston.

\subsection{Ceiling-fan motor noise}

The low-noise design of ceiling fans uses an inside-out motor (Mehta 1991). An inside-out fan motor is a motor with an external rotor as shown in figure 4 . The rotor is heavier than a 
Table 3. The effect of piston cylinder clearances.

\begin{tabular}{lc}
\hline $\begin{array}{l}\text { Piston cylinder } \\
\text { clearance }(\mu \mathrm{m})\end{array}$ & Sound intensity level $(\mathrm{dB})$ \\
\hline 50 & 86.5 \\
33 & 86.4 \\
26 & 79.2 \\
20 & 76.3 \\
\hline
\end{tabular}

conventional motor for the same power with the advantage that it produces a flywheel effect which damps small pole-to-pole magnetic variations. This results in quieter and smoother operation. Ceiling fan motors are usually slow speed (typically 50 to $350 \mathrm{rpm}$ ) and therefore have more poles than usual. The rotor with a larger diameter can have more slots with larger cross-sectional area to reduce power losses and noise. The inside-out motor design has a substantially larger air gap area for the same stator diameter than a conventional motor. This reduces field densities. Motors with higher flux densities can give rise to noise, excessive temperatures and low efficiencies.

\subsection{Cleaning nozzle jet noise}

Jet noise which is at velocities above about $100 \mathrm{~m} / \mathrm{s}$ can be reduced by an extra air stream. This is because the strength of the turbulence is determined by the relationship between the speed of the jet and the speed of the ambient air (Anon 1982). The noise levels can be significantly reduced by introducing an extra air stream of a lower speed along with the jet.

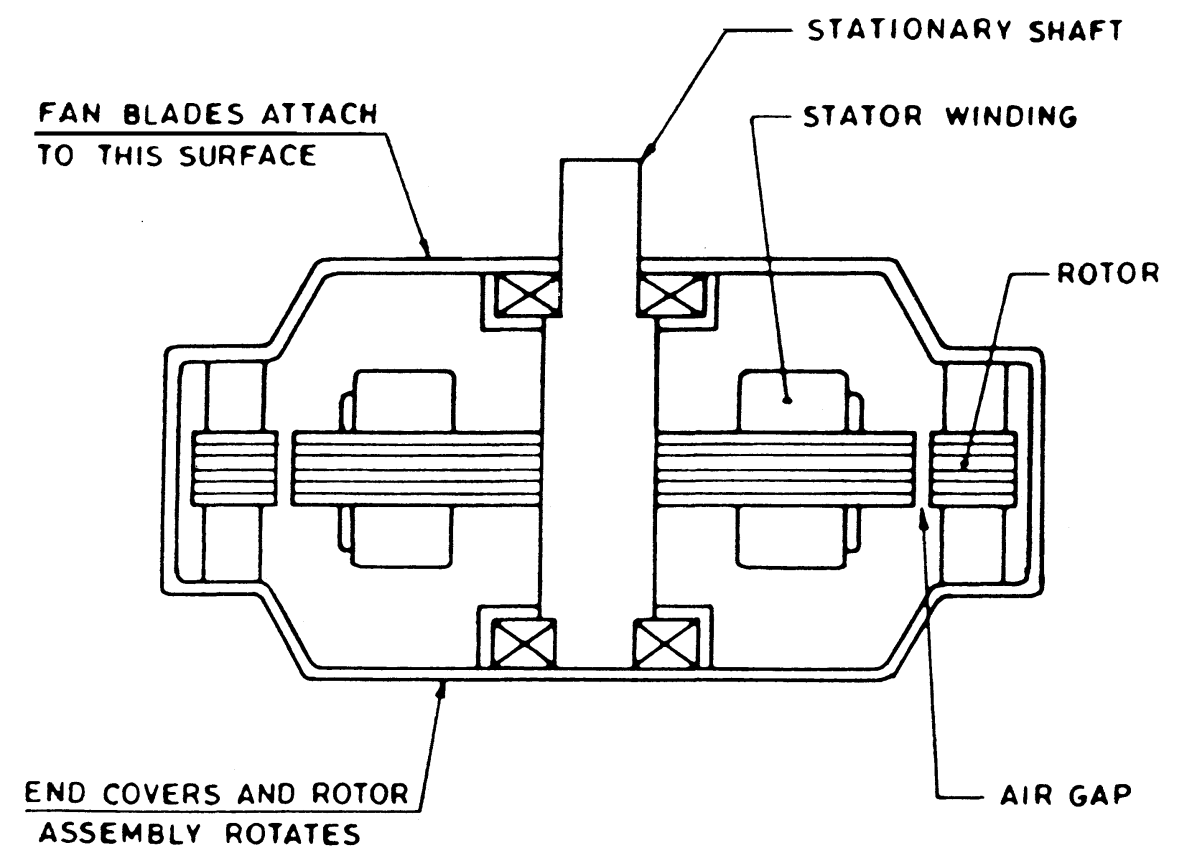

Figure 4. Inside-out ceiling-fan motor. 
This can be employed in the nozzle of the compressed air pipe which is used for cleaning the dirt off machine components in factories. The simple nozzle can be replaced by a compound nozzle which feeds a lower speed annular air stream around the main high speed air stream. The transition from the high speed of the central air stream to still air is much less sudden and the noise level is reduced substantially (Anon 1982).

\section{References}

Anon 1982 Noise control (Naerum, Denmark: Brüel \& Kjaer)

Mehta M 1991 How to drop the ceiling on fan noise. Machine Design 24: 103-106

Rohrle M D 1975 Affecting diesel engine noise by the piston. SAE paper No. 750799: 1985-2001

Tandon N, Nakra B C 1994 Sound intensity technique and noise diagnostics. Eng. Design, J. Natl. Design Res. Forum Inst. Eng. India 25: 25-30

Tandon N, Nakra B C, Sarkar B, Adyanthaya V 1997 Noise control of two-wheeler scooter engine. Appl. Acoust. 51: 369-380

Ubhe D R 1996 Vibration and noise control of engine driven portable generator set. M Tech thesis, Indian Institute of Technology, Delhi

Watts G R, Crombie D H, Hothersall D C 1994 Acoustic performance of new designs of traffic noise. J. Sound Vibr. 177: 289-305 